\title{
Topical issue on Exploring Strongly Interacting Matter at High Densities - NICA White Paper
}

Published online: 29 August 2016 - C Società Italiana di Fisica / Springer-Verlag 2016 \begin{abstract}
presented by NICA, with a particular focus on the following topics:
- phases of dense QCD matter and their possible realization;

- characteristic processes as indicators of phase transformations;

- estimates for events and event rates;

- comparison to other experiments;

- interdisciplinary aspects, e.g. astrophysical constraints for dense matter phases.
\end{abstract}

The aim of the present Topical Issue is to identify the challenges and the discovery potential of heavy-ion collision experiments as those being prepared at the accelerator complex NICA, which will provide the highest baryon densities ever created in terrestrial laboratories. Concerted efforts are required to understand the matter that constitutes the interiors of neutron stars and governs spectacular astrophysical phenomena like supernova explosions and neutron star mergers, which are the most plausible sites for the production of heavy elements in nature. In order to support the construction or modification of heavy-ion collider facilities for experimental studies of QCD phase transformations at high baryon densities, D. Blaschke, A. Sorin and V. Toneev opened in 2009 the "NICA White Paper" forum (http://theor0.jinr.ru/twiki-cgi/view/NICA/WebHome) devoted to developments for the specific opportunities

We especially wish to acknowledge the invaluable encouragement and support of A. Sorin thoughout the various stages of the White Paper leading up to this Topical Issue.

The present edition of this Topical Issue comprises 56 contributions, partly selected and updated from the online White Paper, and partly new contributions fulfilling the condition to address observables to be measured at NICA. Moreover, the introductory section contains five additional contributions describing: 1) the three stages of the contruction of the NICA accelerator complex, 2) the multi-purpose detector (MPD) of the collider experiment, 3) the fixed target experiment for studies of baryonic matter at the nuclotron (BM@N), 4) simulation software for the NICA experiments and 5) the spin physics experiments at NICA-SPD with polarized proton and deuteron beams. Therefore, this Topical Issue shall represent a repository for basic parameters and informations about the NICA facility presently under construction and it shall contribute to shaping its physics programme.

We thank the members of the Program Advisory Committee for Particle Physics at the JINR Dubna for stimulating the development of the NICA White Paper, in particular H. Gutbrod and I. Tserruya. Furthermore, special thanks are due to V. Kekelidze, D. Kharzeev, V. Matveev, A. Sorin, H. Stöcker and Nu Xu. Finally, we wish to dedicate this Topical Issue to the memory of A.N. Sissakian who initiated the establishment of NICA.

David Blaschke, Jörg Aichelin, Elena Bratkovskaya, Volker Friese, Marek Gazdzicki, Jørgen Randrup, Oleg Rogachevsky, Oleg Teryaev, and Viacheslav Toneev 